\title{
EFEITO DA ADIÇÃO DE TERRAS RARAS EM CONJUNTO COM O AGENTE NODULARIZANTE FESIMG NA MICROESTRUTURA E PROPRIEDADES MECÂNICAS DO FERRO FUNDIDO NODULAR J434 D45I 2
}

\author{
Lucas Cunha' \\ Célia de Fraga Malfatti ${ }^{1,2}$
}

Resumo

O objetivo deste trabalho foi avaliar o efeito da adição de duas ligas FeSiMg tipo 4 em diferentes concentrações de terras raras, nomeadas como liga $A$ e $B$, na microestrutura e propriedades mecânicas de um ferro fundido nodular da norma SAE J434 classe D45 I2. Para o desenvolvimento, foi utilizado o mesmo percentual do insumo avaliado em diferentes reações para verificar sua repetibilidade e posteriormente avaliados os corpos de prova de nodularização via microscopia óptica. As propriedades mecânicas foram avaliadas através de ensaios de tração e dureza Brinell. Os resultados mostraram que a liga $\mathrm{A}$ apresentou melhores resultados nas propriedades mecânicas em função no acréscimo de $15 \%$ de perlita na matriz metálica e aumentou o tamanho da grafita esferoidal proporcionado pelo menor percentual de terras raras presente nesta liga.

Palavras-chave: Ferro fundido nodular; Liga FeSiMg tipo 4; J434 D45I2; Terras raras.

\section{EFFECT OF THE ADDITION OF RARE EARTHS IN CONJUNCTION WITH THE NODULARIZING AGENT FESIMG IN THE MICROSTRUCTURE AND MECHANICAL PROPERTIES OF THE DUCTILE CAST IRON J434 D45 I 2}

\begin{abstract}
The objective of this work was to evaluate the effect of the addition of two FeSiMg type 4 alloys at different concentrations of rare earths, named as alloy $A$ and $B$, on the microstructure and mechanical properties of a ductile cast iron SAE J434 class D45 I2. For the development, the same percentage of the evaluated input was used in different reactions to check its repeatability and later the test bodies of nodularization were evaluated by optical microscopy. The mechanical properties were evaluated through tests tensile and hardness Brinell. Results showed that alloy A presented better results in the mechanical properties due to the addition of $15 \%$ of perlite in the metal matrix and increased in the size of the spheroidal graphite provided by the lower percentage of rare earths present in this alloy.
\end{abstract}

Keywords: Ductile cast iron; Alloy FeSiMg type 4; J434 D45 I2; Rare earths.

\section{INTRODUÇÃO}

A utilização do ferro fundido nodular está vinculada à sua ductilidade e resistência mecânica. Essas propriedades permitem a este ferro fundido algumas aplicações em determinados segmentos, tais como, produção de tubos e conexões, peças para indústria automobilística, válvulas e corpos de bombas para indústria do petróleo, componentes de máquinas sujeitos a cargas de choque e fadiga, entre outros. A ductilidade apresentada neste tipo de ferro fundido é proveniente do seu tipo de grafita, na forma esferoidal e com matriz ferrítica é superior quando comparada com a matriz perlítica. Além de possuir ductilidade e resistência mecânica, dependendo da matriz predominante, pode-se favorecer uma das propriedades mencionadas [I,2].

\footnotetext{
'Programa de Pós-graduação em Engenharia Metalúrgica, Faculdade SATC, Criciúma, Santa Catarina, Brasil. E-mail: energiaxmateriais@gmail.com 2Programa de Pós-graduação em Engenharia de Minas, Metalúrgica e de Materiais - PPGE3M, Laboratório de Pesquisa em corrosão - LAPEC, Universidade Federal do Rio Grande do Sul - UFRGS, Farroupilha, Rio Grande do Sul, Brasil.
} 
A obtenção deste ferro fundido depende do processo de nodularização e das condições específicas do metal base, que consiste no tratamento feito no metal base com elementos químicos que favoreçam a formação de grafita esferoidal. As técnicas de nodularização mais conhecidas são, simples transferência, processo sanduíche, panela rotativa e imersão por sino [3,4]. A técnica utilizada neste trabalho baseou-se no uso da panela rotativa, que consiste na adição do elemento nodularizante no reservatório localizado no fundo da panela e não entra em contato com o metal líquido durante seu enchimento, com isso é possível transportar o metal líquido do forno até a linha de vazamento sem que ocorra a reação, permitindo minimizar o fading do agente nodularizante, ou seja, o excesso de perda por volatilização do elemento nodularizante. Entre as matérias-primas utilizadas na fabricação do ferro fundido nodular, principalmente a sucata de aço, pode conter elementos químicos residuais do processo de fabricação ou adicionados propositalmente que são considerados deletérios para grafita esferoidal, por isso é importante a adição de terras raras para neutralizar este efeito indesejável, fornecer um efeito auxiliar ao magnésio para tornar a forma da grafita mais esferoidal e auxilia na nucleação da grafita $[5,6]$.

Com aumento da demanda do mercado de peças que utilizam ligas em ferro fundido nodular de alta resistência mecânica, é necessário utilizar matérias-primas de qualidade para promover tais características, objetivando o menor custo possível.

O presente trabalho tem o objetivo de avaliar o comportamento na microestrutura e propriedades mecânicas na utilização de duas ligas FeSiMg tipo 4 com concentrações diferentes de terras rars na obtenção do ferro fundido nodular da classe D45I 2 da norma J434 [7].

\section{MATERIAIS E MÉTODOS}

As amostras estudadas neste trabalho, foram produzidas no forno de indução sem núcleo com capacidade de $800 \mathrm{~kg}$ do fabricante Inductotherm com potência máxima de $600 \mathrm{~kW}$, utilizando como agente nodularizante a liga de ferro, silício e magnésio do tipo 4 contendo de 5 a $7 \%$ de magnésio, adicionada na panela tratamento conhecida como panela rotativa, conforme ilustrado na Figura I. O processo de tratamento panela rotativa, consiste na adição da liga no reservatório localizado acima do nível de metal líquido para não iniciar a reação antes do tempo desejado, afim de diminuir o fading do agente nodularizante. Para iniciar a reação, é necessário lacrar a tampa da panela, rotacionar para posição vertical e o metal líquido entrará em contato com o agente nodularizante. Após cessado a reação, é transferido o metal para a panela de vazamento e realizado a técnica de inoculação, que consiste na adição de elementos grafitizantes para favorecer a solidificação segundo o sistema estável, evitando-se assim a formação de carbonetos [1-3].

Para o carregamento do forno, primeiramente foi adicionado ferro gusa, pois apresenta menor ponto de fusão, na sequência sucata de aço, grafite sintético e ferro-silício. Com o decorrer da fusão das matérias-primas, foi adicionado retorno até completar toda a capacidade do forno.

A amostra para análise de composição química foi retirada na temperatura mínima de $1450^{\circ} \mathrm{C}$ com o auxílio de um cadinho e vertido em uma coquilha de cobre eletrolítico. A composição química foi mensurada no espectrômetro de emissão óptica (Espectro - modelo Espectromaxx).

O ferro fundido nodular escolhido para o estudo foi da classe D45 I 2 da norma SAE J434 [7], cuja composição química é apresentada na Tabela $I$.

Os valores mínimos e máximos descritos para cada elemento químico são aplicados para todas as classes descritas na referida norma, porém para cada classe é necessário estreitar os valores de acordo com os resultados de resistência à tração, alongamento, dureza e microestrutura, conforme Tabela 2.

Para a obtenção das especificações citadas na Tabela 2, a composição química da Tabela I foi adequada conforme Tabela 3 em função do processo produtivo e suas variáveis.

Após a conformidade com a composição química, - metal líquido foi aquecido para temperatura entre 1500 e $1530^{\circ} \mathrm{C}$ e realizado a limpeza da superfície do metal

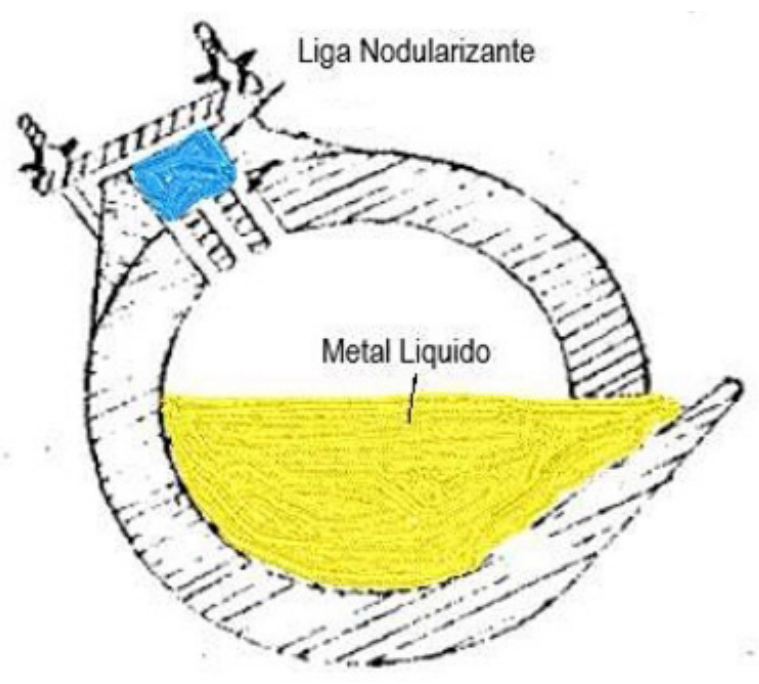

Figura I. Panela rotativa para tratamento de nodularização.

Tabela I. Composição química da classe D45 I2 [7]

\begin{tabular}{ccccccc}
\hline Elementos & $\mathbf{C}$ & $\mathbf{S i}$ & $\mathbf{M n}$ & $\mathbf{P}$ & $\mathbf{S}$ & $\mathbf{M g}$ \\
\hline$\%$ em peso & 3,20 a 4,10 & 1,80 a 3,00 & 0,10 a I,00 & Máx. 0,050 & Máx. 0,035 & 0,025 a 0,060 \\
\hline
\end{tabular}


líquido para retirada de escória decorrente do processo de refino do metal com auxílio do escorificante.

Antes de iniciar o vazamento, a panela de tratamento foi aquecida com maçarico e na sequência foi adicionado a liga FeSiMg tipo 4 com granulometria de 6 a $12 \mathrm{~mm}$ (equivalente a I, $40 \%$ do peso do metal adicionado posteriormente nesta panela). As duas ligas utilizadas apresentam a composição química conforme Tabela 4.

$A$ liga $A$ o percentual de magnésio é superior em sua composição, já percentual de terras raras é superior na liga $B$, entre os elementos dos metais terras raras, destaca-se o cério e lantânio.

Durante a transferência do metal da panela de tratamento para a panela de vazamento, foi adicionado $0,40 \%$ de inoculante contendo silíco, cálcio e bário realizado no jato de metal entre as panelas. Durante o vazamento é realizado a medição da temperatura do metal com auxílio do pirômetro de imersão da marca Italtherm utilizando

Tabela 2. Especificações da classe D45 I 2 [7]

\begin{tabular}{lc}
\hline \multicolumn{1}{c}{ Ensaio } & Valores \\
\hline Resistência a tração mínima & $450 \mathrm{Mpa}$ \\
Alongamento mínimo & \multicolumn{1}{c}{$\mathrm{I} \%$} \\
Dureza & $\mathrm{I} 56$ a $217 \mathrm{HBW}$ \\
Microestrutura & $\begin{array}{l}\text { 80\% de grafita do tipo I e II com } \\
\text { matriz ferrítica-perlítica e isenta } \\
\text { de carbonetos }\end{array}$ \\
\hline
\end{tabular}

o sensor do tipo " $S$ " para manter-se em média a $1400^{\circ} \mathrm{C}$ evitando qualquer tipo de defeito metalúrgico. $O$ tempo de resfriamento de 30 minutos foi mantido para todas as amostras.

Em cada panela de tratamento, foram vazados quatro blocos em " $Y$ " para retirada de corpo de prova de tração indicado na região I, amostra para medição de dureza superficial e amostra para ensaio metalográfico indicados nas regiões 2 conforme Figura $2[8,9]$.

Para a avaliar se o percentual dos elementos terras raras resultantes serão 0 suficiente para neutralizar 0 efeito deletério dos elementos $\mathrm{Ti}, \mathrm{As}, \mathrm{Sn}, \mathrm{Sb}, \mathrm{Pb}, \mathrm{Bi}$ e Al na morfologia da grafita esferoidal, será utilizado o fator Thielman K, conforme Equação I, onde recomenda-se para valores menores que 0,80 o percentual máximo de terras raras deve ser $0,010 \%$, mas sua adição não é obrigatória. Já para valores de 0,80 a 2,00 a adição de terras raras é obrigatória, porém em excesso causa a degeneração da grafita e formação de carbonetos $[5,6,10]$.

$$
\begin{aligned}
& K=4,4 \times T i+2,0 \times A s+2,3 \times S n+5,0 \times \\
& S b+290,0 \times P b+370,0 \times B i+1,6 \times A l
\end{aligned}
$$

A medição da dureza superficial foi realizada no durômetro de bancada do fabricante Time Group Inc. modelo TH600 com esfera de carbeto de tungstênio de diâmetro de $5 \mathrm{~mm}$ com carga aplicada de $750 \mathrm{kgf}$ durante 10s, foi realizado 3 identações por amostra [I I].

Tabela 3. Composição química da classe D45 I 2 final [7]

\begin{tabular}{ccccccc}
\hline Elementos & $\mathbf{C}$ & $\mathbf{S i}$ & $\mathbf{M n}$ & $\mathbf{P}$ & $\mathbf{S}$ & $\mathbf{M g}$ \\
\hline \% em peso & 3,40 a 3,80 & 2,30 a 2,80 & 0,30 a 0,60 & Max. 0,050 & Max. 0,035 & 0,025 a 0,060 \\
\hline
\end{tabular}

Tabela 4. Composição química das ligas

\begin{tabular}{cccccc}
\hline Elementos (\% em peso) & Si & Mg & Ca & Terras Raras & Al \\
\hline Liga A & 45,50 & 7,00 & 1,50 & $0,5 \mathrm{I}$ & 0,50 \\
Liga B & 46,00 & 6,05 & 0,96 & 1,20 & 0,63 \\
\hline
\end{tabular}
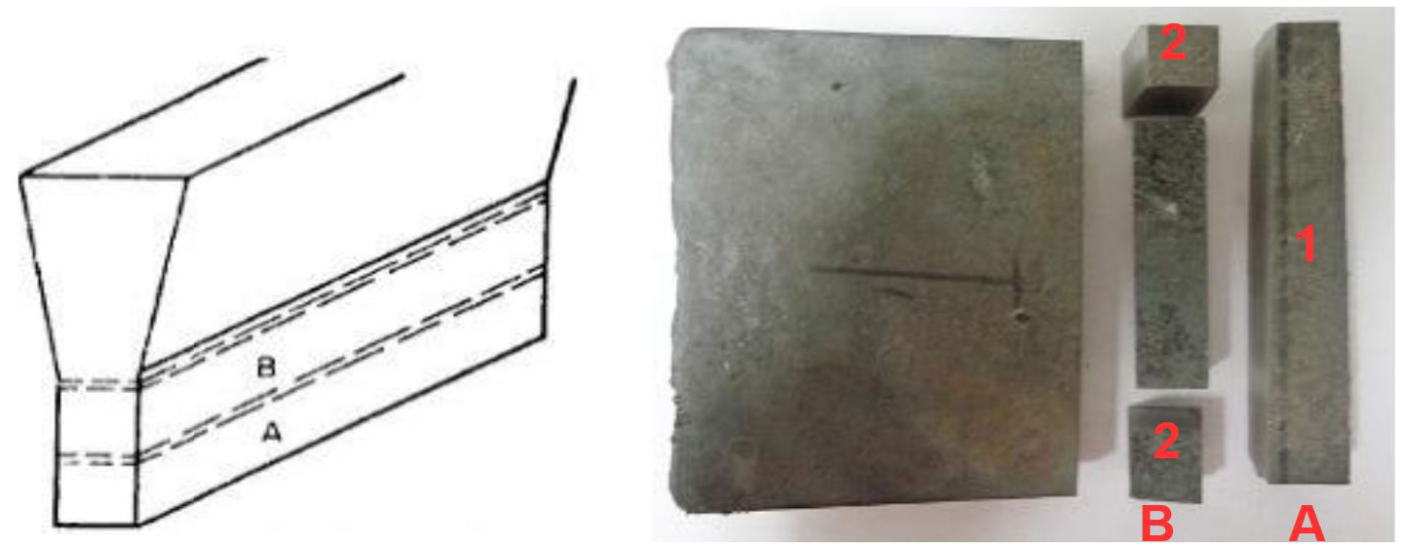

Figura 2. Local de retirada dos corpos de provas no bloco Y. 
Para realização dos testes de tração utilizou-se uma máquina de tração universal da fabricante EMIC com capacidade de 20 toneladas de carga utilizando o corpo de prova de acordo com a norma [8].

Para análise metalográfica as amostras foram cortadas, lixadas e polidas com suspensão de alumina I $\mu \mathrm{m}$ e posteriormente atacadas com nital $2 \%$. As microestruturas foram avaliadas em um microscópio óptico (Olympus - mod. UC30) e classificados segundo as normas SAE J434 [7], ASTM E8 [9], ASTM A247 [12] e ABNT NBR 6593-8I [13].

\section{RESULTADOS E DISCUSSÃO}

A composição química do metal antes de realizar a adição das ligas e inoculante, ou seja, o metal base do forno esta apresentado na Tabela 5.

A composição química das amostras após o tratamento de nodularização e a técnica de inoculação produzidas com a adição da liga A e B, estão ilustrados nas Tabelas 6 e 7.

Conforme Tabelas 6 e 7, verifica-se o efeito dessulfurante do magnésio, pois o percentual de enxofre resultante após o tratamento de nodularização é inferior ao metal base em todas adições, os resultados obtidos estão de acordo com o indicado pela literatura, pode variar de 0,005 a 0,008\% [14, I5]. Ainda é possível verificar o acréscimo de silício em todas as adições proveniente da adição de liga FeSiMg e do inoculante. Para ambas adições o fator de Thielman $\mathrm{K}$ foi igual a I,03, para liga $\mathrm{A}$ o percentual de terras raras, ou seja, o somatório de Ce e La foi de 0,009\% e para liga $B$ o percentual de terras raras obtido foi de $0,014 \%$.

As microestruturas evidenciam, conforme Figuras 3 e 4 , para ambas as ligas a morfologia da grafita na forma esferoidal, já a liga $A$ apresentou um acréscimo de $15 \%$ de perlita na matriz metálica em relação a liga $B$.

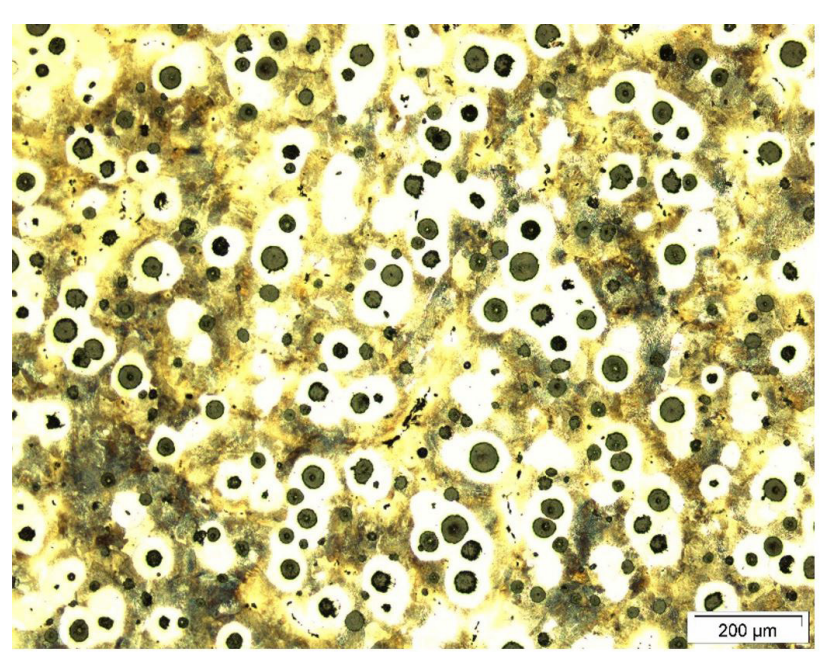

Figura 3. Metalografia da amostra com a liga A.

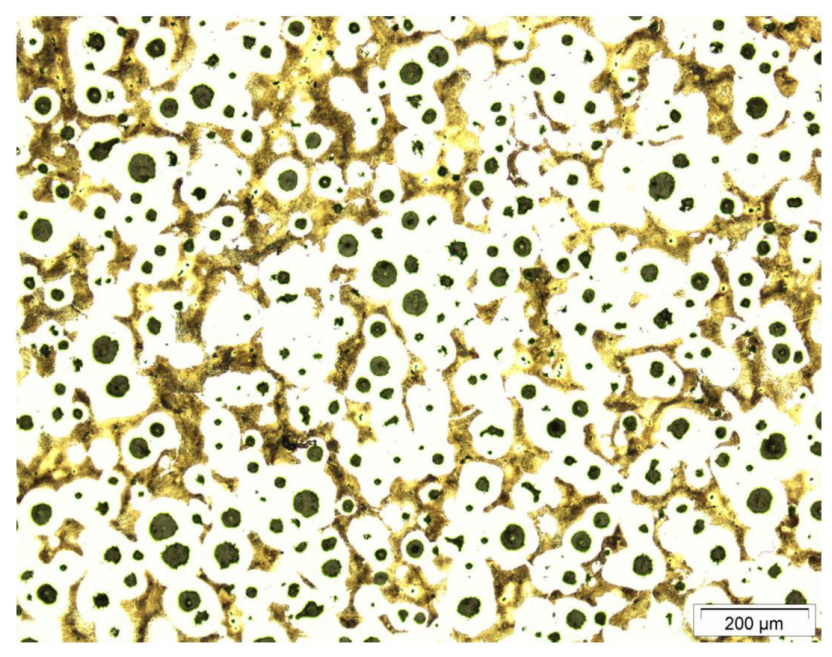

Figura 4. Metalografia da amostra com a liga B.

Tabela 5. Composição química do metal base no forno

\begin{tabular}{cccccccccccc}
\hline Elementos & $\mathbf{C}$ & $\mathbf{S i}$ & $\mathbf{M n}$ & $\mathbf{P}$ & $\mathbf{S}$ & $\mathbf{M g}$ & $\mathbf{C e}$ & $\mathbf{L a}$ & $\mathbf{T i}$ & $\mathbf{A s}$ \\
\hline \% em peso & 3,62 & $\mathrm{I}, 56$ & 0,53 & 0,016 & 0,014 & $<0,00 \mathrm{I}$ & $<0,003$ & $<0,00 \mathrm{I}$ & 0,008 & 0,004 \\
\hline Elementos & $\mathbf{S n}$ & $\mathbf{S b}$ & $\mathbf{P b}$ & $\mathbf{B i}$ & $\mathbf{A l}$ & $\mathbf{C r}$ & $\mathbf{C u}$ & $\mathbf{M o}$ & $\mathbf{V}$ & $\mathbf{F e}$ \\
\hline$\%$ em peso & 0,007 & $0,00 \mathrm{I}$ & 0,002 & $0,00 \mathrm{I}$ & $0,0 \mathrm{II}$ & 0,038 & 0,085 & $0,00 \mathrm{I}$ & 0,004 & $\mathrm{Bal}$. \\
\hline
\end{tabular}

Tabela 6. Composição química das amostras com adição da liga $A$

\begin{tabular}{cccccccccccc}
\hline Elementos & $\mathbf{C}$ & $\mathbf{S i}$ & $\mathbf{M n}$ & $\mathbf{P}$ & $\mathbf{S}$ & $\mathbf{M g}$ & $\mathbf{C e}$ & $\mathbf{L a}$ & $\mathbf{T i}$ & $\mathbf{A s}$ \\
\hline \% em peso & 3,58 & 2,65 & 0,54 & 0,017 & 0,007 & 0,044 & 0,006 & 0,003 & 0,008 & 0,004 \\
\hline Elementos & $\mathbf{S n}$ & $\mathbf{S b}$ & $\mathbf{P b}$ & $\mathbf{B i}$ & $\mathbf{A l}$ & $\mathbf{C r}$ & $\mathbf{C u}$ & $\mathbf{M o}$ & $\mathbf{V}$ & $\mathbf{F e}$ \\
\hline \% em peso & 0,007 & $0,00 \mathrm{I}$ & 0,002 & 0,001 & 0,012 & 0,037 & 0,083 & 0,001 & 0,003 & $\mathrm{Bal}$ \\
\hline
\end{tabular}

Tabela 7. Composição química das amostras com adição da liga $B$

\begin{tabular}{cccccccccccc}
\hline Elementos & $\mathbf{C}$ & $\mathbf{S i}$ & $\mathbf{M n}$ & $\mathbf{P}$ & $\mathbf{S}$ & $\mathbf{M g}$ & $\mathbf{C e}$ & $\mathbf{L a}$ & $\mathbf{T i}$ & $\mathbf{A s}$ \\
\hline \% em peso & 3,59 & 2,67 & 0,54 & 0,016 & 0,007 & $0,04 \mathrm{I}$ & 0,009 & 0,005 & 0,008 & 0,004 \\
\hline Elementos & $\mathbf{S n}$ & $\mathbf{S b}$ & $\mathbf{P b}$ & $\mathbf{B i}$ & $\mathbf{A l}$ & $\mathbf{C r}$ & $\mathbf{C u}$ & $\mathbf{M o}$ & $\mathbf{V}$ & $\mathbf{F e}$ \\
\hline$\%$ em peso & 0,007 & $0,00 \mathrm{I}$ & 0,002 & 0,001 & 0,012 & 0,037 & 0,084 & 0,001 & 0,004 & $\mathrm{Bal}$. \\
\hline
\end{tabular}


Tabela 8. Morfologia da grafita e propriedades mecânicas das amostras da liga A

\begin{tabular}{|c|c|c|c|c|c|}
\hline Dados & Nodularização (\%) & Nódulos $/ \mathrm{mm}^{2}$ & $\begin{array}{c}\text { Dureza Brinell } \\
\text { (HB) }\end{array}$ & $\begin{array}{l}\text { Resistência à } \\
\text { Tração (MPa) }\end{array}$ & Alongamento (\%) \\
\hline Média & 94,2 & 145,2 & 199,5 & 505,5 & 12,8 \\
\hline Desvio Padrão & 0,9 & 8,5 & 5,0 & $\mathrm{II}, 4$ & 0,5 \\
\hline
\end{tabular}

Tabela 9. Morfologia da grafita e propriedades mecânicas das amostras da liga B

\begin{tabular}{lccccc}
\hline \multicolumn{1}{c}{ Dados } & Nodularização (\%) & Nódulos $/ \mathbf{m m}^{2}$ & $\begin{array}{c}\text { Dureza Brinell } \\
\text { (HB) }\end{array}$ & $\begin{array}{r}\text { Resistência à } \\
\text { Tração (MPa) }\end{array}$ & $\begin{array}{c}\text { Alongamento (\%) } \\
\text { Média }\end{array}$ \\
Desvio Padrão & 96,2 & 175,2 & 175,0 & 469,5 & 15,6 \\
\hline
\end{tabular}
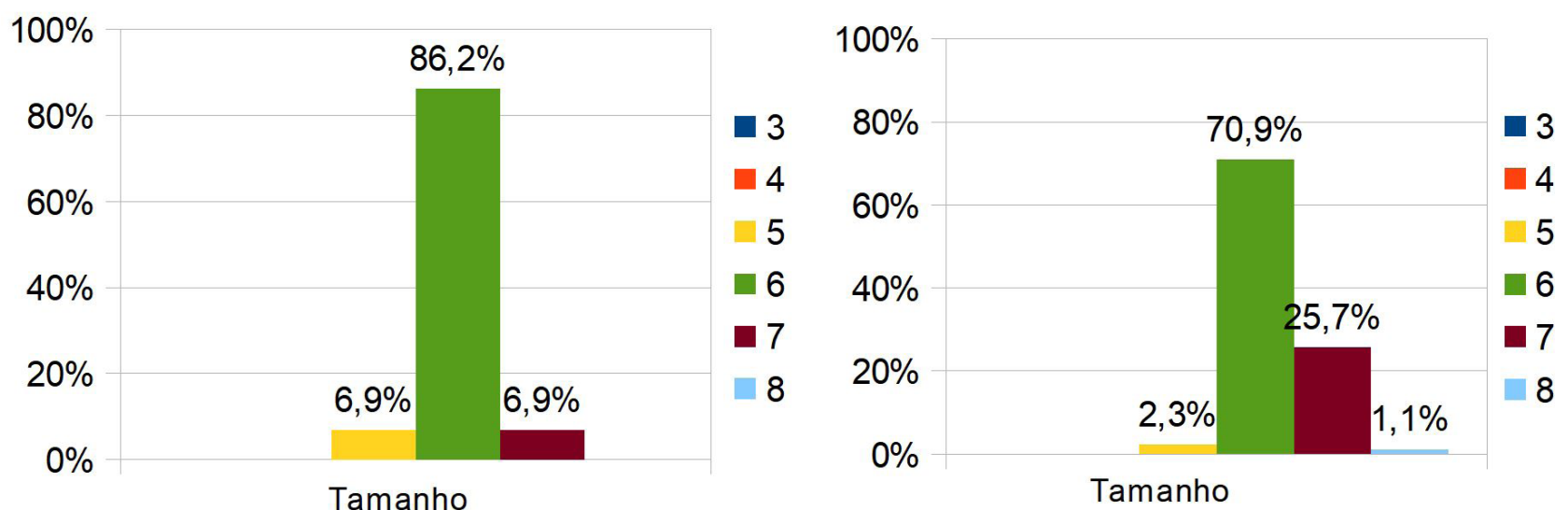

Figura 5. Distribuição do tamanho da grafita da amostra com a liga $A$ (esquerda) e com a liga $B$ (direita).

Já a Figura 5, as amostras produzidas com liga A, o tamanho médio da grafita resultante foi 6 e para as amostras da liga $B \circ$ tamanho médio da grafita resultante foi entre 6 e 7.

Para o fator K resultante de I,03, os valores de terras raras de ambas adições foi o suficiente para neutralizar os elementos que degeneram a grafita e auxiliou o magnésio a torna a grafita mais esferoidal, já o acréscimo de $35 \%$ obtido nas amostras da liga $B$ de terras raras, auxiliou na nucleação aumentando o número de nódulos por unidade de área, reduzindo seu tamanho e tornar o superesfriamento mais intenso, pois outras variáveis que afetam a quantidade de nódulos por unidade de área foram mantidas constantes, entre elas a principal é o processo de inoculação $[2,16]$. Este acréscimo de terras raras nas amostras da liga $B$ não foi o suficiente para promover a degeneração da grafita e formar carbonetos conforme citado $[5,6]$.

Ainda em relação as Tabelas 8 e 9, é possível observar que houve uma pequena variação de dureza entre as ligas testadas. A liga A o valor resultante foi $10 \%$ superior em função do acréscimo de perlita na matriz metálica [16]. Esse acréscimo de perlita ainda promoveu um ganho de $3 \%$ na resistência à tração e reduziu o alongamento em $10 \%$.

\section{CONCLUSÃO}

Todos os resultados obtidos das amostras produzidas com a liga $A$ e B apresentam conformidade com a classe D45 I 2 da norma J434.

O percentual obtido em todas as amostras de magnésio e terras raras promoveram o grau de nodularização mínimo de $93 \%$, em função do efeito nodularizante.

A amostra com a liga $A$ apresentou $15 \%$ de acréscimo de perlita na matriz metálica que favoreceu um acréscimo de $10 \%$ na dureza Brinell e $3 \%$ na resistência à tração, porém um decréscimo de $10 \%$ no alongamento. Em função do efeito nucleante das terras raras, a liga $\mathrm{A}$ em função do seu menor percentual desses elementos, favoreceu uma redução no número de nódulos por unidade de área e o aumento do tamanho da grafita.

O percentual resultante de terras raras nas amostras de ambas as ligas, promoveram a neutralização dos elementos deletérios da grafita esferoidal e o acréscimo de $35 \%$ de terras raras presente nas amostras da liga B não foi o suficiente para degenerar a grafita esferoidal e precipitar carbonetos. 


\section{REFERÊNCIAS}

I Chiaverini V. Aços e ferros fundidos. 7. ed. São Paulo: ABM; 1996.

2 Santos ABS. Castello branco: metalurgia dos ferros fundidos cinzentos e nodulares. São Paulo, IPT; 1977.

3 Vilela, FJ. Efeito de algumas variáveis de processo na obtenção do ferro fundido nodular ferritico no estado bruto de fundição [dissertação]. São Caetano do Sul: Instituto Mauá de Tecnologia; 2010.

4 Santos A. B. S, Beckert E. A, Fenilli R, Pieske A. et al. Processos de nodularização de ferros fundidos. Metalúrgia ABM. 1983;39:52I-526.

5 Riposan I, Chisamera M, Uta, V, Stan S, Naro R, Willians D. et al. The importance of rare earth contribution from nodulizing alloys and subsequent effect on the inoculation of ductile iron. Romania: Politehnica University of Bucharest; 2014.

6 Uta V, Riposan I. Structural characteristics of ductile iron at different rare earth contribution from a magnesium ferrosilicon alloys. Romania: Politehnica University Bucharest; 2014.

7 SAE International. SAE J434 - automotive ductible iron castings. Michigan: SAE International; 2004.

8 American Society for Testing and Materials. ASTM E8 - standart test method for tension test of metallic materials. West Conshohocken: ASTM Intenational; 2010.

9 American Society for Testing And Materials. ASTM A370 - Standart test methods and definitions for mechanical testing of steel products. West Conshohocken: ASTM International; 2003.

I0 Pan F, Zhang J, Chen H-L, Su Y-H, Kuo C-L, Su Y-H et al. Effects of rare earths metals on steel microstructures. Materials (Basel); 9(6):4I7.

II American Society for Testing and Materials. ASTM EIO - standart test method for Brinell hardness of metallic materials. West Conshohocken: ASTM International; 2004.

12 American Society for Testing and Materials. ASTM A247 - standart test method for evaluating the microestruture of grafite in iron castings. West Conshohocken: ASTM International; 1998.

13 Associação Brasileira de Normas Técnicas. ABNT NBR 6593-8I- morfologia de grafita em ferro fundido. Rio de Janeiro: ABNT; 198I.

I4 Riposan I, Chisamera M, Kelley R, Barstow M, Naro RL. Magnesium-sulfur relationships in ductile an d compacted cast irons as influenced by late sulfur additions. AFS Transsactions 2003;93: I- I5.

I5 Naidek VL, Verkhovliuk AM. Some considerations of the mechanism of nodular graphite formation in cast iron. Kyiv, Ukraine: National Academy of Sciences of Ukraine; 2015.

16 Melo GHT, Carmo DJ. Correlação entre a microestrutura e propriedades mecânicas do ferro fundido com grafita esferoidal. Itaúna: SENAI/CETEF; 1992. 36 p.

Recebido em: II Maio 2018

Aceito em: 2 Maio 2019 